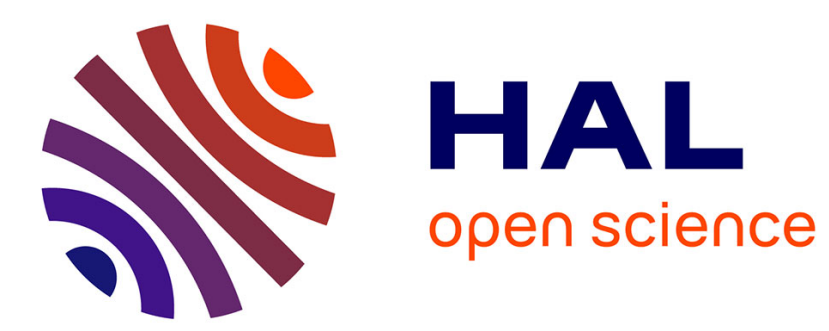

\title{
Instability of polymer films
}

\author{
A. Halperin, P. Pincus, S. Alexander
}

\section{To cite this version:}

A. Halperin, P. Pincus, S. Alexander. Instability of polymer films. Journal de Physique Lettres, 1985, 46 (12), pp.543-547. 10.1051/jphyslet:019850046012054300 . jpa-00232557

\section{HAL Id: jpa-00232557 https://hal.science/jpa-00232557}

Submitted on 1 Jan 1985

HAL is a multi-disciplinary open access archive for the deposit and dissemination of scientific research documents, whether they are published or not. The documents may come from teaching and research institutions in France or abroad, or from public or private research centers.
L'archive ouverte pluridisciplinaire HAL, est destinée au dépôt et à la diffusion de documents scientifiques de niveau recherche, publiés ou non, émanant des établissements d'enseignement et de recherche français ou étrangers, des laboratoires publics ou privés. 
Classification

Physics Abstracts

$68.15-82.65 \mathrm{~F}$

\title{
Instability of polymer films
}

\author{
A. Halperin, P. Pincus and S. Alexander (*) \\ Exxon Research and Engineering Company, Annandale, New Jersey, U.S.A.
}

(Reçu le 4 mars 1985, accepté le 19 avril 1985)

\begin{abstract}
Résumé. - Nous étudions un ensemble dilué de chaînes polymériques confinées dans un film de solvant étalé sur une surface solide. Nous montrons que, pour un faible mouillage, il existe une interaction attractive entre chaînes qui peut conduire à une séparation de phase bidimensionnelle, même en régime de bon solvant.
\end{abstract}

\begin{abstract}
We consider a dilute collection of polymer chains confined to a solvent film which is spread upon a solid surface. We show that for weak wetting there exist a long range attractive interaction between chains which may lead to a two dimensional phase separation even in good solvents.
\end{abstract}

\section{Introduction.}

The spreading of a fluid on a solid surface has many practical applications to coating formation, permeation in porous media, etc. Recently Joanny and de Gennes [1] have considered the balance between interfacial tension and Van der Waals forces to determine the equilibrium thickness $h$ and shape of a wetting film. Here, we consider the slightly more complicated case "here the spreading fluid is a good solvent for polymer chains. In particular, if the bulk solution polymer radius of gyration $R_{\mathrm{g}}$ exceeds the film thickness, $h$, we expect a shape deformation of the layer. The principal point of the present work is to show that this perturbation may be long range and lead to a polymer demixtion even for good solvents.

The tendency for a fluid to wet a solid surface is determined [1] by the spreading coefficient $S$,

$$
S=\gamma_{\mathrm{SV}}-\left(\gamma+\gamma_{\mathrm{SL}}\right)
$$

where $\gamma_{\mathrm{Sv}}, \gamma, \gamma_{\mathrm{SL}}$ are respectively the solid-vapour, liquid-vapour, and solid-liquid surface tensions. For $S>0$, a finite contact angle is impossible and the solvent is said to wet the surface. Then, for the case of a " dry " solvent [2], the equilibrium thickness of the wetting film is usually given by the balance between the spreading force and the long range Van der Waals interaction which may tend to favour thick films [3]. This interaction may be written in the form

$$
P(h)=\frac{1}{2} \gamma(a / h)^{2}
$$

(*) Permanent address : Racah Institute of Physics, Hebrew University, Jerusalem, Israel. 
for non-retarded interactions between parallel surfaces separated by a distance $h . \gamma a^{2}$ is proportional to the Hamaker constant which fixes $a$ to be an atomic dimension $(\sim 1 \AA)$. Neglecting edge effects, the free energy of the film is

$$
f=f_{0}+A[P(h)-S],
$$

where $A$ is the film area. Minimizing the free energy with respect to $h$ at constant volume, $V=A h$, yields the equilibrium film thickness [1] $h_{0}$,

$$
h_{0}=a(3 \gamma / 2 S)^{1 / 2} \text {. }
$$

For most practical situations with finite $S, h_{0}$ is less than about $100 \AA$.

For $R_{\mathrm{g}}>h_{0}$, a polymer chain dissolved in the solvent will perturb the film leading to a profile (Fig. 1) $h(\mathbf{r})$ where $\mathbf{r}$ is the in-plane coordinate. The shape of the bulge is determined from the free energy [1]

$$
f-f_{0}=\int\left[-S+P(h)+\frac{1}{2} \gamma(\nabla h)^{2}+\mu h+\Lambda(h, \mathbf{r})\right] \mathrm{d}^{2} r
$$

where $\mu$ is a Lagrange multiplier assuring the constant volume constraint and $\Lambda(h, r)$ is the polymer induced perturbation. For a localized perturbation, it is straightforward to verify the

$$
\mu=\gamma a^{2} / h_{0}^{3} .
$$

Defining $y=h / h_{0}, \rho=\sqrt{3} r / \lambda, \lambda=h_{0}^{2} / a,(1.5)$ becomes

$$
\left(2 / \gamma h_{0}^{2}\right)\left(f-f_{0}\right)=\int\left[G(y)+\left(\nabla_{\rho} y\right)^{2}+\tilde{\Lambda}(y, \rho)\right] \mathrm{d}^{2} \rho,
$$

where $G(y)=\frac{1}{3}\left(y^{-2}+2 y-3\right)$. With the free energy functional expressed in this dimensionless form, the healing length associated with a film deformation is clearly $\lambda$ For $h_{0} \sim 100 \AA$, $\lambda \sim 10^{3}-10^{4} \AA$ which may exceed the in-plane polymer radius of gyration. For good solvents, an isolated polymer chain confined to a film of thickness $h$ has a transverse radius [4] given by

$$
R_{\mathrm{F}} \cong N^{3 / 4}(b / h)^{1 / 4} b
$$

where $b$ is a typical monomer dimension and $N$ is the degree of polymerization. For $N \sim 10^{4}$, (1.8) yields $R_{\mathrm{F}} \lessgtr 10^{3} \AA$, and we find two possible cases $\lambda>R_{\mathrm{F}}$ or $\lambda<\boldsymbol{R}_{\mathrm{F}}$ even for $\boldsymbol{R}_{\mathrm{F}}>\boldsymbol{h}_{\mathbf{0}}$.

In the next section, we concentrate on the situation $\lambda>R_{F}>h_{0}$ and demonstrate that at finite but dilute polymer concentrations, phase separation is probable.

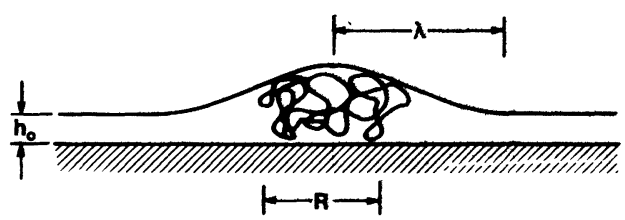

Fig. 1. - Sketch of the film with the polymer generated distortion. 


\section{Demixtion.}

For $\lambda \gg R_{\mathrm{F}}$, the polymer acts like a localized potential on the film. For distances from the polymer $r>R_{\mathrm{F}}$, the Euler-Lagrange equation which minimizes (1.7) is

$$
\nabla_{\rho}^{2} y=\frac{1}{3}\left(1-y^{-3}\right)
$$

which may be linearized $(y=1+\psi)$ to yield

$$
\nabla_{\rho}^{2} \psi=\psi .
$$

The solution which tends to zero at infinity is the Bessel function of an imaginary argument $\psi=\psi_{0} K_{0}(\rho)$ where asymptotically $\psi \rightarrow(\pi / 2 \rho)^{1 / 2} \mathrm{e}^{-\rho}$ for $\rho \rightarrow \infty$, and $\psi_{0}$ is an arbitrary amplitude. Near the origin $\psi$ diverges as $-\ln \rho$. Because $\psi(\rho)$ is slowly varying for small arguments, it may be approximately taken to be constant over the region occupied by the polymer coil. Then the free energy (1.7) becomes

$$
\left(f-f_{0}\right)\left(2 / \gamma h_{0}^{2}\right) \cong 2 \pi \int\left[\psi^{2}+(\nabla \psi)^{2}\right] \rho \mathrm{d} \rho+\left(2 / \gamma h_{0}^{2}\right) f_{\mathbf{P}}
$$

where the good solvent polymer confinement energy $f_{\mathrm{P}}$ is [5]

$$
f_{\mathrm{P}} \cong N T\left(b / h_{\mathrm{m}}\right)^{5 / 3}
$$

and $h_{\mathrm{m}}$ is the amplitude on the deformation near the origin, i.e. $h_{\mathrm{m}} \approx h_{0}\left(1-\psi_{0} \ln \rho_{0}\right)$ and $\rho_{0}=\sqrt{3} R_{\mathrm{F}} / \lambda$. Minimizing (2.4) with respect to the amplitude $\psi_{0}$, we find

$$
\psi_{0} \cong K \sigma\left(R_{\mathrm{F}} / \lambda\right)^{4 / 3}(a / \lambda)^{1 / 3}
$$

where $R_{\mathrm{F}}$ is given by (1.8), the polymer-film coupling constant $\sigma$ is $\sigma=\left(2 T / \gamma a^{2}\right) \sim 1$, and $K$ is a constant of order unity [6].

The long range interaction $U(r)$ between two coils separated by a distance $r \gg R_{\mathrm{F}}$ arises from one chain lowering its confinement energy (2.4) by taking advantage of the long range tail of the bulge deformation created by the other chain, i.e.

$$
U_{i}(r) \cong-\frac{5}{3} N T\left(a / h_{0}\right)^{5 / 3} \psi(r) .
$$

When two chains overlap there is a strong repulsion leading to an effective net interaction $U(r)$,

$$
U(r)=\left\{\begin{array}{ll}
\infty & r<R_{\mathrm{F}} \\
U_{i}(r) & r>R_{\mathrm{F}}
\end{array} .\right.
$$

In terms of $U(r)$, the two dimension second virial coefficient $V$ between polymer coils is

$$
V=\int\left[1-\mathrm{e}^{-U(\boldsymbol{r}) / T}\right] \mathrm{d}^{2} r .
$$

For weak coupling $\left(\psi_{0}<1\right), V$ is approximately

$$
V \cong \pi R_{\mathrm{F}}^{2}-(2 \pi / 3) \lambda^{2} \sigma\left(R_{\mathrm{F}} \lambda\right)^{8 / 3}(\lambda / a)^{1 / 3} .
$$

For a negative second virial we expect a phase separation whenever $|V| / R_{\mathrm{F}}^{2}>\phi^{-1}$, where $\phi$ 
is the average fraction of the film area occupied by chains. This would occur at a critical coupling constant $\sigma_{\mathrm{cr}}=\sigma^{*} / \phi$

$$
\sigma^{*} \approx\left(\lambda a / R_{\mathrm{F}}^{2}\right)^{1 / 3}=\left(h_{0} / R_{\mathrm{F}}\right)^{2 / 3}<1 .
$$

The dense phase is composed of touching polymer chains with a corresponding monomer area fraction $c^{*} \approx N^{-1 / 2}\left(h_{0} / a\right)^{1 / 2} \ll 1$. The thickness of the film in this region relative to $h_{0}$ will be $\delta h / h_{0} \approx \sigma \sigma^{*}$. The dilute phase will be a film of thickness $h_{0}$ with a concentration of chains approximately $\sigma^{*} / \sigma$.

Thus for a given coupling constant $\sigma$, the biphasic domain should cover the range $1>\phi>\sigma^{*} / \sigma$. For $\phi>1$, the chains overlap and interpenetrate forming a semi-dilute solution. The film will increasingly thicken and become smoother.

\section{Final remarks.}

We have shown that for weakly spreading films $\left(h_{0} \gg a\right)$, the long range attractive interaction between dissolved polymer coils may induce a two dimensional phase separation. What is the situation for $\lambda<R_{\mathbf{F}}$, i.e. either small chains or stronger spreading coefficient ? In this case, the bulge is restricted to the area where the polymer is localized. This results in a lateral pressure tending to collapse the chain. Approximating the bulge by a rectangular deformation of amplitude $\delta h$ and surface area $R^{2}$, the change of free energy associated with the presence of the polymer is

$$
\delta f \cong \frac{3}{2} \pi \gamma R^{2}\left(a / h_{0}\right)^{2}\left(\delta h / h_{0}\right)^{2}+2 \pi R \gamma \delta h+f_{\mathrm{P}} .
$$

The first term is the cost in free energy in deforming the film away from the wetting-Van der Waals balance, the second term is the surface tension increase arising from the increased liquidvapour surface area and the final term, $f_{\mathrm{P}}$ is the polymer confinement energy which tends to thicken the film. This latter term differs from (2.4) in that one should allow for lateral compression which might develop to optimize the first two terms in (3.1),

$$
f_{\mathrm{P}} / T \cong N(b / h)^{5 / 3}+\left(R_{\mathrm{F}} / R\right)^{4} .
$$

The second term which is the increase of excluded volume and elastic energy in compressing a two dimensional swollen chain may be determined by a blob analysis [7] given in the Appendix.

The expression (3.1) should now be optimized both with respect to $\delta h$ and $R$. Neglecting the surface tension term (which may be verified as small $a$ posteriori), we find

$$
\begin{gathered}
\delta h / h_{0} \approx \sigma^{2} ; \\
R \approx(N / \sigma)^{1 / 2}\left(h_{0} / a\right)^{1 / 0} a
\end{gathered}
$$

for weak coupling, $\sigma<1$. The lateral pressure contracts the polymer toward a collapsed chain of $h$ blobs for $\sigma \simeq 1$. For a finite (but dilute) density of coils, the neglected surface tension provides the attractive interaction between chains leading to phase separation.

Further investigations should consider other polymer-surface coupling, e.g. attractive interactions, and the dynamics of the demixtion.

\section{Acknowledgments.}

We are pleased to thank P. G. de Gennes, J. F. Joanny, F. Rondelez, S. Garoff and M. Alvarez for helpful discussions. 


\section{Appendix.}

A polymer chain constrained to two dimensions in a good solvent is swollen to a radius given by [4]

$$
R_{2} \cong N^{3 / 4} a
$$

What is the free energy $\delta f$ required to isotropically compress the coil to a radius $R<R_{2}$ ? A blob analysis [7] is now given to obtain the result used in (3.2), i.e. $\delta f / T \approx\left(R_{2} / R\right)^{4}$. Consider the compressed polymer as a chain of blobs of radius $\xi$, where on a short length scale $r<\xi$, the two dimensional structure is preserved. Then the average monomer concentration $c=N / R^{2}$ may be expressed as $c=g / \xi^{2}$ where the number of monomers $g$ per blobs is given by

$$
\xi \simeq g^{3 / 4} a .
$$

These expressions yield $g \approx\left(c a^{2}\right)^{-2}$. The free energy of deformation is then $T /$ blob or

$$
\begin{aligned}
\delta f & =T(N / g) \cong N T\left(c a^{2}\right)^{2} \\
& =N T\left(N a^{2} / R^{2}\right)^{2}
\end{aligned}
$$

Using (A.1) we find

$$
\delta f \cong T\left(R_{2} / R\right)^{4} .
$$

In this derivation we have neglected the confinement energy arising from a chain of blobs which would yield an additional contribution proportional to $R^{-2}$. Above four dimensions this term would dominate.

\section{References}

[1] Joanny, J. F. and De Gennes, P. G., C. R. Hebd. Séan. Acad. Sci. Paris, Sér. II 299 (1984) 279.

[2] By " dry » fluid, we mean a low vapour pressure liquid where the volume of the film or drop may be considered to be a conserved quantity.

[3] De Gennes, P. G., C. R. Hebd. Séan. Acad. Sci. Paris, Sér. II 297 (1983) 9.

[4] See for example, De Gennes, P. G., Revista Del Nuovo Cimento 7 (1977) 363.

[5] De GenNes, P. G., Macromolecules 14 (1981) 1637. This expression results from scaling arguments and is only correct to a numerical factor of order unity.

[6] For simplicity we have taken $b=a$. The constant $K$ actually has a weak logarithmic dependence on $\rho_{0}$.

[7] For a general reference see De Gennes, P. G., Scaling Concepts in Polymer Physics (Cornell Press, Ithaca) 1979. Here again numerical coefficients of order unity have been dropped. 